\section{Light driven reactions of single physisorbed azobenzenes $\dagger$}

\author{
Maciej Bazarnik, ${ }^{* a}$ Jörg Henzl, ${ }^{b}$ Ryszard Czajka ${ }^{a}$ and Karina Morgenstern ${ }^{b}$ \\ Received 18th March 2011, Accepted 13th May 2011 \\ DOI: $10.1039 / \mathrm{c} 1 \mathrm{cc11578b}$
}

We present a successful attempt of decoupling a dye molecule from a metallic surface via physisorption for enabling direct photoisomerization. Effective switching between the isomers is possible by exposure to UV light via the rotation pathway.

Functionalization of metal and semiconductor surfaces with molecular switches is an emerging trend in nanotechnology aimed at nanoelectronic applications with recently renewed interest due to improved experimental possibilities. Switches should be both reversible and bistable. To fulfill these requirements a molecule must have at least two different thermally stable and distinguishable forms and a possibility of repeatedly triggering externally an interconversion between them. Since isomers often differ significantly in their physical and chemical properties, isomerization might serve as a molecular switching mechanism. Azobenzene and its derivatives are perfect prototypes for photochromic molecular switches, because they isomerise between an elongated trans and a compact cis isomer with a strong change in conductivity. Therefore, they have been studied widely on surfaces. ${ }^{1-9}$ There are reports of cis-trans isomerization induced by tunneling electrons, ${ }^{6-8}$ by the electric field in the tip-surface junction, ${ }^{4}$ or by photons. ${ }^{1-3}$ Most of the experiments were performed on the $\mathrm{Au}(111)$ surface, ${ }^{1-4,6,9}$ which is well known for its weak interaction with adsorbed molecules. Until recently, reversible isomerization on more reactive surfaces, such as $\mathrm{Cu}$ substrates, was generally believed to be impossible even when triggered by electrons. ${ }^{6}$ Recent studies demonstrated the switching of molecules adsorbed on $\mathrm{Cu}$ or even $\mathrm{Si}^{5,10}$ However, the chemisorption of the molecules alter the isomers such that the switch gets conformational rather than configurational.

Photoisomerization is unlikely for molecules adsorbed on metals because of alternative faster excitation channels. Indeed, the photoisomerization of native azobenzene molecules directly adsorbed on a metal substrate is suppressed ${ }^{1}$ presumably because of a shorter lifetime of the photo-excited state on metals. As a consequence the azobenzene molecules were functionalized with tert-butyl spacer groups, which are intended to lift the

\footnotetext{
${ }^{a}$ Institute of Physics, Poznan University of Technology,

Nieszawska 13A, 60-965 Poznan, Poland.

E-mail: Maciej.Bazarnik@put.poznan.pl; Fax: +48 61665 3178;

Tel: + 48616653194

${ }^{b}$ Division of Atomic and Molecular Structures (ATMOS),

Leibniz University of Hannover, Appelstr. 2, D-30167 Hannover, Germany

$\dagger$ Electronic supplementary information (ESI) available: Details of structure calculations. See DOI: 10.1039/c1cc11578b
}

azobenzene scaffold away from the surface. Though exposure to light led to isomerization of this TBA (tert-butyl azobenzene) on $\mathrm{Au}(111),{ }^{1,2}$ the light is adsorbed by the metal's d-band. ${ }^{3}$ The hole created in this way relaxes to the band top to initiate the isomerization via electron transfer from the highest occupied molecular orbital (HOMO) to the hole. The direct photoisomerization based on absorbance of the light by the molecule has not been achieved yet for supported molecules.

Moreover, it is likely that molecular switches will be incorporated into already existing technologies based on silicon instead of being adsorbed on a non-inert surface like $\mathrm{Au}(111)$. It was shown that slightly more reactive surfaces suppress electroninduced isomerization, e.g. TBA on $\mathrm{Cu}(111)$ and $\mathrm{Au}(100){ }^{6}$ Furthermore, surface mediated photoisomerization will not work with a different d-band structure of the substrate. Here, we propose to use molecules trapped in a physisorption well. ${ }^{11}$ This opens the way for direct photo-switching of adsorbed molecules.

The 4 -anilino- $4^{\prime}$-nitroazobenzene molecule ${ }^{12}$ is functionalized to be trapped in its physisorption well by three aromatic carbon rings and three nitrogen containing groups (Fig. 1a and $b$ ). The three nitrogen containing groups are expected to pull the molecule towards the surface, and the three aromatic rings, named 1,2 , and 3 , to counteract this attraction by trying to establish a van-der-Waals distance. ${ }^{8}$ As long as the molecule is physisorbed its electronic structure is hardly perturbed from its gas phase counterpart, therefore there is little reason for the molecule to change its configuration on the large scale. However, configurational changes with very small changes in energy as the rotation around a single bond are possible upon adsorption. In Fig. 1a and $b$ both isomers are shown in the gas phase as calculated on the basis of density functional theory with slight adjustments for the adsorption (see ESI $\dagger$ ). The trans isomer is elongated and virtually two-dimensional, while the cis form is twisted, with all three rings bent out-of-plane.

The experiments are carried out in ultra-high vacuum at $6 \mathrm{~K}$ by means of a low-temperature scanning tunneling microscope $\left(\mathrm{STM}\right.$, Createc $\left.^{\mathrm{TM}}\right)$. A $\mathrm{Cu}(111)$ surface is cleaned by standard sputtering and annealing cycles. Next, the molecules are deposited on the substrate kept at (a) $20 \mathrm{~K}$ and (b) $100 \mathrm{~K}$. In both cases two predominant shapes are observed, consistent with the size and shape of the simulated molecules (Fig. 1c and d). On the basis of structural analysis and electrostatic potential (ESP) maps ${ }^{13}$ we identify the triangular form with three protrusions (Fig. 1c) as the cis isomer, and the more elongated form with two protrusions (Fig. 1d) as the trans isomer. 


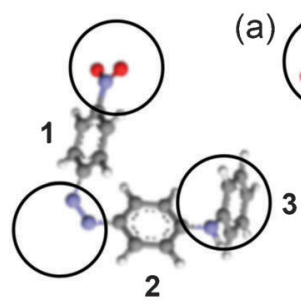

2

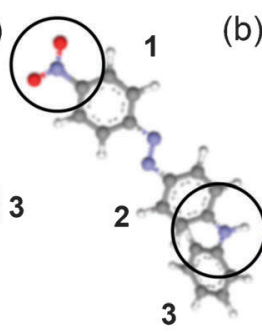

(b)

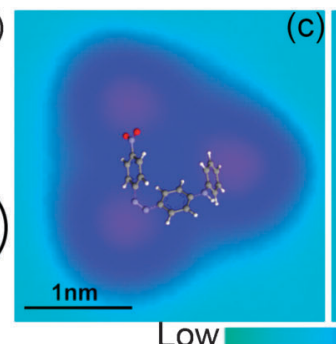

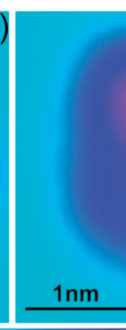

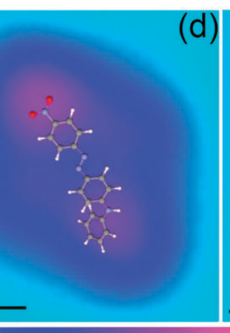

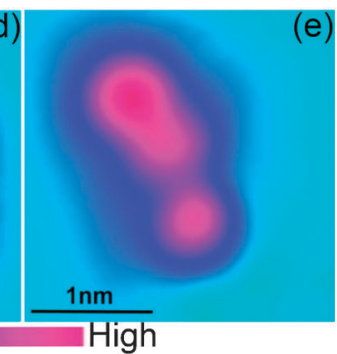

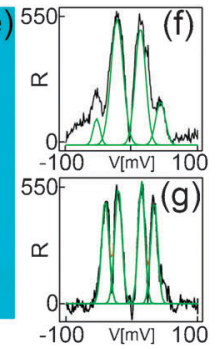

Fig. 1 4-Anilino-4'-nitroazobenzene: (a) cis-isomer and (b) trans-isomer calculated in the gas phase by DFT geometry optimization (see ESI $\dagger$ for details); circles in gray, blue, red, and light gray represent $\mathrm{C}, \mathrm{N}, \mathrm{O}$, and $\mathrm{H}$ atoms, respectively; black circles indicate regions of largest negative density; numbers identify rings (c-e) STM images superimposed with ball-and-stick models scaled 2:1 against models in (a) and (b); (c) physisorbed cis-isomer; (d) physisorbed trans-isomer; (e) chemisorbed form; tunneling parameters: (c) $43 \mathrm{pA}, 50 \mathrm{mV}$, (d) $69 \mathrm{pA}, 50 \mathrm{mV}$, (e) $69 \mathrm{pA}$, $100 \mathrm{mV}$; (f, g) IETS spectra of (c) and (d); green curves are Gaussian fits to the peaks; $R$ stands for $R$-value of $\mathrm{d}^{2} I / \mathrm{d}^{2}\left[\mathrm{nA} \mathrm{V} \mathrm{V}^{-2}\right]$; lock-in parameters: $f_{\text {mod }}=334 \mathrm{~Hz}, V_{\text {mod }}=20 \mathrm{mV}$.

To further verify our assignment we analyzed the vibrational structure of the two forms as measured in inelastic electron tunneling spectroscopy (IETS) in a lock-in technique. ${ }^{14}$ Because of electron induced changes to the molecules at higher voltages, we are thereby restricted to low energy modes. Though these are not expected to differ in energy for the two isomers, we expect different efficiencies of the modes because of the different configuration of the molecular groups with respect to the tunneling current direction. In the measured energy range, the IET spectra of both forms reveal two peaks at $\sim 20 \mathrm{meV}$ and at $\sim 50 \mathrm{meV}$ (Fig. If and g). The peak at $20 \mathrm{meV}$ represents the vibration of the whole molecule with respect to the $\mathrm{Cu}(111)$ surface. The peak at $50 \mathrm{meV}$ represents an internal vibration of the molecule. Out of several possible vibrations the ring out-of-plane deformation is most likely. ${ }^{15}$ We performed position dependent IETS measurements but show only representative spectra taken above the middle of the molecule because they show both peaks with mean value and can be used to compare both species. The spectra of cis and trans isomers differ in the width of the $20 \mathrm{meV}$ peak (full width at half maximum: $20 \mathrm{meV}$ on cis vs. $14 \mathrm{meV}$ on trans at the same $V_{\text {mod }}$ ) and in the intensity of the $50 \mathrm{meV}$ peak, which changes by $60 \%$. The broader $20 \mathrm{meV}$ peak indicates a superposition of several energetically similar vibrations. This is consistent with the three-dimensionality of the cis form as opposed to the two-dimensional trans form, giving rise to more and less possibilities of vibrations against the surface, respectively. To explain the $60 \%$ difference in intensity of the $50 \mathrm{meV}$ peak we employ the propensity rules for the IETS proposed by Troisi and Ratner. ${ }^{16}$ These rules state that the most intense IET modes are associated with those vibrations that most effectively modulate the overall coupling among orbitals in contact with the electrodes (in our case surface and tip). Therefore, vibrations that occur in the direction perpendicular to the surface are those that modulate the transmission of the electrons through the molecule most. The two isomers differ in the angle between the carbon rings and the surface (Fig. 1a and b). Thus, the component of the out-of-plane ring deformation vibration perpendicular to the surface differs. Consequently, the influence of this vibration on the modulation of the electron transition through the molecule is smaller for the cis-form than for the trans-form. The changes in IETS spectra thus support our image interpretation. The close resemblance to the gas-phase molecule suggests physisorption.
The physisorption of the molecules is confirmed by their conversion into the chemisorbed form by a local high voltagehigh current treatment (for details see ESI $\dagger$ ). The treatment affects an area of about $500 \mu \mathrm{m}$ in radius. In the middle of this area all molecules are desorbed. Near its border molecules have converted into a linear shape consisting of three protrusions, two very close ones and one slightly detached (Fig. 1e). This structure resembles closely the appearance of 4-anilino$4^{\prime}$-nitroazobeneze adsorbed on $\mathrm{Au}(111)$ at $250 \mathrm{~K},{ }^{9}$ i.e. the chemisorbed form on another metal surface. As local heating cannot explain the chemisorption, a non-adiabatic transition induced by high current is more probable. The chemisorbed form does not isomerize on $\mathrm{Cu}(111)$ under electron irradiation, at least not with a yield higher than $10^{-10}$ per electron.

Final conformation of physisorption is the yield of electroninduced isomerization (discussed in detail below) of the order of $10^{-7}$ per electron, which is an order of magnitude higher than that obtained for chemisorbed azobenzene derivatives. ${ }^{7} \mathrm{We}$ have thus established that two isomers of anilino-nitroazobenzene physisorb on the $\mathrm{Cu}(111)$ surface at low temperature. Lightinduced excitation might live long enough on these weakly bound molecules to trigger isomerization.

We now irradiate the sample surface in the STM by light from a $100 \mathrm{~W}$ mercury lamp which has a rich spectrum, ranging from $250 \mathrm{~nm}$ to $600 \mathrm{~nm}$ and fully covering the range of the cis-trans-isomerization related absorption of 4-anilino4'-nitroazobenzene (Fig. 2d) (for details see ESI $\dagger$ ). During irradiation the temperature of the sample rises to $16 \mathrm{~K}$. A control experiment, in which the sample is annealed in situ to $16 \mathrm{~K}$ for 30 minutes, excludes this temperature rise to be responsible for the processes induced.

The large-scale images in Fig. 2 clearly show the same region of the surface before and after irradiation. In the small-scale images a molecule is seen to reversibly switch from the cis form to the trans form and back as a result of the irradiation. In this particular case, only one molecule was seen to isomerize in the observed area. Overall, more than 600 molecules were exposed to the full spectrum of the lamp $\left(1.1 \mathrm{~W} \mathrm{~cm}^{-2}\right.$ on the sample) for 20 minutes. This corresponds to a photon dose of $1 \times 10^{20} \mathrm{~cm}^{2}$. 23 molecules ( $4 \%$ of the population) on the surface isomerize, and $3(0.5 \%$ of the population) undergo an apparent rotation (discussed below). 


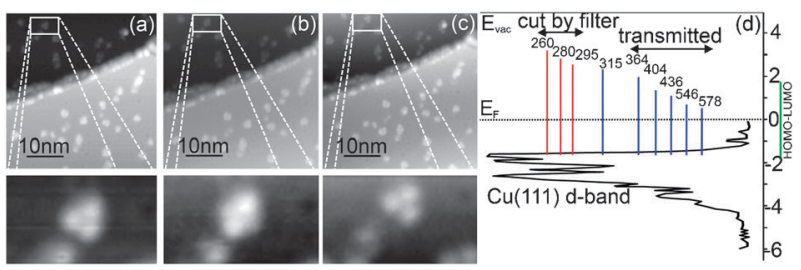

Fig. 2 Light-induced isomerization: STM images before (a), after first (b), and after second (c) irradiation with enlargement as indicated; $56 \mathrm{pA}, 52 \mathrm{mV}$; (d) schematic representation of the energy overlap of the $\mathrm{Cu}(111)$ d-band, the mercury lamp emission, the BG-39 filter transmittance, and the 4-anilino-4'-nitroazobenzene HOMO-LUMO gap; note that positions of the HOMO and LUMO are schematic only.

As discussed in the introduction, light-induced isomerization of TBA molecules on $\mathrm{Au}(111)$ is substrate mediated, ${ }^{3}$ involving the photo-excitation of d-band electrons. To verify that the process observed in our experiment is not substrate mediated, we use a BG-39 filter. This filter transmits photons in the energy range from $330 \mathrm{~nm}$ to $600 \mathrm{~nm}$, only. The $\mathrm{Hg}$ lamp emits at the wavelengths sketched in Fig. 2 d lines of a width of $\sim 2 \mathrm{~nm}$. The filter blocks the wavelengths $260 \mathrm{~nm}, 280 \mathrm{~nm}$ and 290-300 nm completely, the line around $315 \mathrm{~nm}$ is reduced to $40 \%$. All other lines are transmitted by $90 \%$. Using the filter, no isomerization or rotation is observed. This excludes all wavelengths above $315 \mathrm{~nm}$ to be responsible for the isomerization shown in Fig. $2 \mathrm{a}-\mathrm{c}$. As the position of the d-bands of $\mathrm{Cu}(111)$ is less than $2 \mathrm{eV}$ below the Fermi energy (Fig. 2d), the substrate mediated process is excluded as it should be possible also with wavelengths transmitted by the filter that have energies between $3.76 \mathrm{eV}$ and $2.07 \mathrm{eV}$. In the gas phase photoisomerization is induced by the transition of an electron either from the HOMO or the HOMO - 1 orbital to the LUMO orbital corresponding to an $\mathrm{n}-\mathrm{pi}^{*}$ and a pi-pi* transition, respectively. For 4-anilino-4'-nitroazobenzene the band related to the $\mathrm{n}$-pi* has its absorption maximum at $435.5 \mathrm{~nm}(2.85 \mathrm{eV})$ and the pi-pi* transition at $332 \mathrm{~nm}(3.73 \mathrm{eV}) .{ }^{17}$ Our filter experiment shows that isomerization based on the n-pi* transition is quenched on the surface. The photoisomerization is thus initiated by the absorption of light by the absorption band covering $220 \mathrm{~nm}$ upto $350 \mathrm{~nm}$ and connected to the pi-pi* transition. Note that this transition is followed by the rotation pathway of the isomerization around the double $\mathrm{N}$ bond of the azo group. We have also conducted a flashphotolysis experiment in acetonitrile solution using a $284 \mathrm{~nm}$ laser confirming our interpretation (to be published elsewhere).

Finally, the observed apparent rotation was investigated in detail by inelastic electron tunneling (IET) manipulation in the range from $150 \mathrm{mV}$ to $600 \mathrm{mV}$ (for details see ESI $\dagger$ ). Two series out of 300 are shown in Fig. 3. Series (a) shows an apparent rotation by $60^{\circ}$ of the molecule around its central point. Only angles representing multiples of $60^{\circ}$ are observed in this process. Series (b) shows the reversible isomerization from cis to trans and back to cis. Comparison of the first and the last image in series (b) with the second and the third image in series (a) suggests that the apparent rotation is a multiple isomerization. Indeed, changes in the $I(t)$ characteristics during manipulation are very similar for the two processes. We do not observe any dependence of the outcome of the manipulation

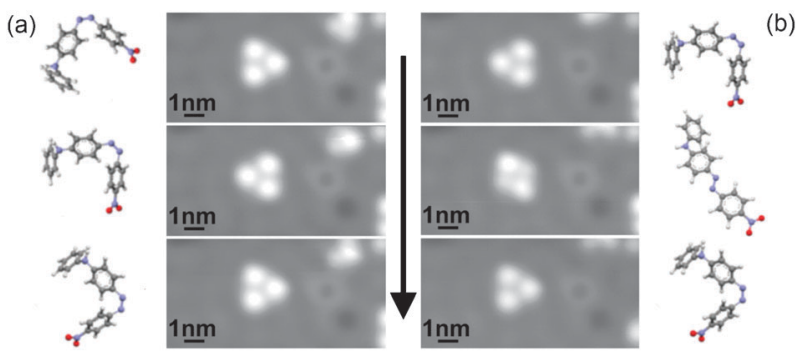

Fig. 3 IET manipulation: (a) apparent rotation (b) isomerization; tunneling parameters: $69 \mathrm{pA}, 100 \mathrm{mV}$, manipulation parameters: $230 \mathrm{mV}, 69 \mathrm{pA}, 5 \mathrm{~s}$. Ball-and-stick models represent configuration of molecules.

on the tip position above the molecule. Note that reversible IET induced isomerization of azobenzene derivatives on surfaces more reactive than $\mathrm{Au}(111)$ has not been reported yet.

Counting the apparent rotation as double isomerization and considering that those photons that are transmitted by the BG-39 filter do not contribute to the isomerization, we calculate that the total effective cross section is $6 \times 10^{-20} \mathrm{~cm}^{-2}$. This is an order of magnitude higher than for surface mediated isomerization of TBA adsorbed on $\mathrm{Au}(111){ }^{18}$

In conclusion, electron-induced and direct photoisomerization of a dye molecule are feasible even on a noninert surface such as $\mathrm{Cu}(111)$. Decoupling is achieved via physisorption. In order to build similar molecular photo-switches on other surfaces a molecule with an appropriate balance of surface pulling and pushing groups depending on the strength of the interactions between the groups and the surface needs to be synthesized. On a metal surface, pi-systems are good candidates for pushing groups and sulfur containing groups might work equally well as nitrogen containing groups for pulling.

MB acknowledges support from the COST Action CM0601, Electron Controlled Chemical Lithography (ECCL) for a short term scientific mission to Hannover.

\section{Notes and references}

1 M. Comstock, et al., Phys. Rev. Lett., 2007, 99, 038301.

2 S. Hagen, F. Leyssner, D. Nandi, M. Wolf and P. Tegeder, Chem. Phys. Lett., 2007, 444, 85.

3 S. Hagen, et al., J. Chem. Phys., 2008, 129, 164102.

4 M. Alemani, et al., J. Am. Chem. Soc., 2006, 128, 14446.

5 N. Henningsen, R. Rurali, K. Franke, I. Fernandez-Torrente and J. Pascual, Appl. Phys. A: Mater. Sci. Process., 2008, 93, 241.

6 M. Alemani, et al., J. Phys. Chem. C, 2008, 112, 10509.

7 K. Morgenstern, Acc. Chem. Res., 2009, 42, 213.

8 E. McNellis, J. Meyer, D. A. Baghi and K. Reuter, Phys. Rev. B: Condens. Matter Mater. Phys., 2009, 80, 35414.

9 J. Henzl and K. Morgenstern, Phys. Chem. Chem. Phys., 2010, 12, 6035 .

10 D. Riedel, et al., J. Am. Chem. Soc., 2009, 131, 5414.

11 D. E. Brown, D. J. Moffatt and R. A. Wolkow, Science, 1998, 279, 542 .

12 S. Kobayashi, H. Yokoyama and H. Kamei, Chem. Phys. Lett., 1987, 138, 333.

13 H. Gawronski, J. Henzl, V. Simic-Milosevic and K. Morgenstern, Appl. Surf. Sci., 2007, 253, 9047.

14 W. Ho, J. Chem. Phys., 2002, 117, 11033.

15 N. Biswas and S. Umapathy, J. Phys. Chem. A, 2000, 104, 2734.

16 A. Troisi and M. A. Ratner, J. Chem. Phys., 2006, 125, 214709.

17 S. R. Hair, G. A. Taylor and L. W. Schultz, J. Chem. Educ., 1990, 67, 709 .

18 M. J. Comstock, et al., Appl. Phys. Lett., 2008, 92, 123107. 